\section{COLLAPSE OF DETERMINISM}

A

$\mathrm{T}$ the thirty-first meeting of the Indian Science Congress, which met in Delhi in January 1944, the Congress president, Prof. S. N. Bose, discussed "The Classical Determinism and the Quantum Theory", showing how "physicists have gained knowledge but lost their faith". This was supplemented in the Section of Mathematics and Statistics by B. M. Sen, president of that Section, in his address entitled "The Fundamental Equations of Quantum Mechanies". It is difficult to do justice to this without mathematical symbols, so the following article will be based mainly on Prof. Bose's more general treatment.

Classical physies may be said to have begun with Newton. His laws of motion and theory of gravitation gave an explanation of planetary motion which was so satisfactory that it seemed to provide an infallible means of predicting the motion of the solar system. Laplace went so far as to assert that if a sufficiently vast intellect knew the mass, position and velocity of every particle of the universe at any one instant, and the forces acting on them, then "nothing would be uncertain for him; the future as well as the past would be present to his eyes".

It was difficult to fit the phenomena of light into this scheme, since the discovery of interference had shown that light moves like waves rather than like particles. But Maxwell overcame this difficulty by developing Faraday's ideas about the ether. Newton's absolute space was no longer to be regarded as empty, but as a medium possessing energy and momentum, capable of being strained and transmitting waves. This theory was later extended to explain also X-rays, radio signals, and the $\gamma$-rays emitted by radium. Maxwell's electromagnetic theory was extended by Lorentz into a theory of electrons. The modifications in the Newtonian scheme had left it stronger than before. Almost everywhere was seen the reign of exact laws. It was true that in thermodynamics physicists had to make shift with laws that applied only to large aggregates, but no one doubted that these were derivable by averaging from exact equations which were too numerous to be conveniently considered individually. The derivation of these averages contained the term probability or chance, but it was always pointed out that really there was no such thing, and that every occurrence could be predicted if all the circumstances were known. It was characteristic of the eighteenth century, the 'age of reason', that Voltaire should assert that "Chance is a word void of sense : nothing can exist without a cause", and Linnæus that "Nature does not proceed by jumps".

These beliefs were rudely shaken by the quantum theory. In 1900 Planck, puzzled by the phenomena of heat radiation, made the revolutionary suggestion that the emission of energy is discontinuous. Soon afterwards Einstein made a similar suggestion about the photo-electric effect. Bohr explained the spectra of hydrogen and other atoms by postulating that an electron must pass discontinuously from one orbit to another. These postulates were empirical, put forward so as to lead up to the results of experiment. They succeeded in this, but at the cost of abandoning the established laws of mechanics and electrodynamics. What seemed a greater break with tradition was called for by the theory of relativity, but in fact this theory does not reject causality. It is true that it denies the existence of absolute time and absolute space, but it sets up a new set of abso. lute laws in space-time which are independent of all axes of reference. Einstein's success in explaining the slight discrepancy in the motion of the planet Mercury, and in predicting the apparent displacement of stars during a solar eclipse, struck the imagination of the public, but really it is quantum theory that clashes fundamentally with classical physics.

At one time there were hopes of a reconciliation through Schrödinger's wave mechanics, but it turned out that the waves in question are only mathematical fictions. They are excellent as a means of calculation, as they can be treated by the familiar methods of differential equations instead of by the unfamiliar matrices of Heisenberg or Dirac's algebra of observ. ables. However, all three methods are equivalent, and all lead to the same startling conclusion, Heisenberg's Principle of Uncertainty. This shows that if we measure the position and momentum of a stream of electrons, the more accurately we determine the position the less accurately can we determine the momentum, and conversely. Some think that this merely expresses the obvious truth that every experiment interferes to some extent with the phenomena we attempt to measure. Others go further, and interpret the principle to mean that we cannot predict the motion of a single particle exactly, owing to the slight variation of the forces on it caused by atomic structure, although we can obtain statistical laws which hold for the average motion of a large number of particles. But a third interpretation goes so far as to claim that the existence of causality is disproved. Von Neumann claims to have demonstrated that the results of the quantum theory cannot be obtained by averaging any exact causal laws.

Some philosopher-physicists welcome these conclusions, as giving us a hope of escape from the tyranny of an iron law of causation, and assuring freewill to mankind as well as to electrons! However, the majority of physicists regard causality as essential to science, and are hoping to establish a unified theory that will once again reunite all our knowledge into exact causal laws. While the matter is still unsettled, we should do well not to insist on imposing our preconceived ideas upon Nature, but let them evolve and adjust themselves to our growing, if somewhat unmanageable, knowledge of reality.

$$
\text { H. T. H. Piaggio. }
$$

\section{THYROXINE: ITS BIOSYNTHESIS AND ITS IMMUNO-CHEMISTRY*}

$\mathrm{T}$

HE suggestion that thyroxine might be formed in Nature from tyrosine through the stage of diiodotyrosine was made at an early stage of the elucidation of the chemistry of thyroxine, and was made more probable when the constitution of the latter was finally determined. Over a number of years several pieces of evidence, all indirect in character, were brought forward in support of this biogenetic hypothesis, which thus came to be generally aecepted.

Ricently two lines of direct evidence have become available which seem to place the matter beyond doubt. In the first place, the transformation of diiodotyrosine into thyroxine has been effected by purely chemical methods of a character which make

* Abstract of the Croonian Lecture of the Royal Society delivered by Dr. C. R. Harington, F.R.S., on July 13. 